\title{
Ab-initio Green's Functions Calculations of Atoms
}

\author{
C. Barbieri* and D. Van Neck ${ }^{\dagger}$ \\ ${ }^{*}$ Theoretical Nuclear Physics Laboratory, RIKEN Nishina Center, 2-1 Hirosawa, Wako, Saitama \\ 351-0198 Japan \\ ${ }^{\dagger}$ Center for Molecular Modeling, Ghent University, Proeftuinstraat 86, B-9000 Gent, Belgium
}

\begin{abstract}
The Faddeev random phase approximation (FRPA) method is applied to calculate the ground state and ionization energies of simple atoms. First ionization energies agree with the experiment at the level of $\sim 10 \mathrm{mH}$ or less. Calculations with similar accuracy are expected to provide information required for developing the proposed quasiparticle-DFT method.
\end{abstract}

Keywords: Green's functions theory; ab-initio quantum chemistry; ionization energies

PACS: $31.10 .+\mathrm{z}, 31.15$.Ar

Introduction. Ab initio treatments of electronic systems become unworkable for sufficiently complex systems. On the other hand, the Kohn-Sham formulation [1] of density functional theory (DFT) [2] incorporates many-body correlations (beyond HartreeFock), while only single-particle (sp) equations must be solved. Due to this simplicity DFT is the only feasible approach in some modern applications of electronic structure theory. There is therefore a continuing interest in studying conceptual improvements and extensions to the DFT framework.

An approach in this direction has been proposed in Ref. [3] by developing a quasiparticle (QP)-DFT formalism. In the QP-DFT the full spectral function is decomposed in the contribution of the QP excitations, and a remainder or background part. It is sufficient to have a functional model for the energy-averaged background part to set up a singleelectron self-consistency problem that generates the QP excitations. Such an approach is appealing since it contains the well-developed standard Kohn-Sham formulation of DFT as a special case, while at the same time emphasis is put on the correct description of QPs, in the Landau-Migdal sense [4]. Hence, it can provide an improved description of the dynamics at the Fermi surface. Given the close relation between QP-DFT and the Green's functions (GF) formulation of many-body theory [5, 6], it is natural to employ ab initio calculations in the latter formalism to investigate the structure of possible QPDFT functionals. This talk reports on recent advances in such calculations.

The Faddeev-RPA (FRPA) method. The theoretical framework of the present study is that of propagator theory, where the object of interest is the sp propagator [5, 6],

$$
g_{\alpha \beta}(\omega)=\sum_{n} \frac{\left\langle\Psi_{0}^{N}\left|c_{\alpha}\right| \Psi_{n}^{N+1}\right\rangle\left\langle\Psi_{n}^{N+1}\left|c_{\beta}^{\dagger}\right| \Psi_{0}^{N}\right\rangle}{\omega-\left(E_{n}^{N+1}-E_{0}^{N}\right)+i \eta}+\sum_{k} \frac{\left\langle\Psi_{0}^{N}\left|c_{\beta}^{\dagger}\right| \Psi_{k}^{N-1}\right\rangle\left\langle\Psi_{k}^{N-1}\left|c_{\alpha}\right| \Psi_{0}^{N}\right\rangle}{\omega-\left(E_{0}^{N}-E_{k}^{N-1}\right)-i \eta}
$$



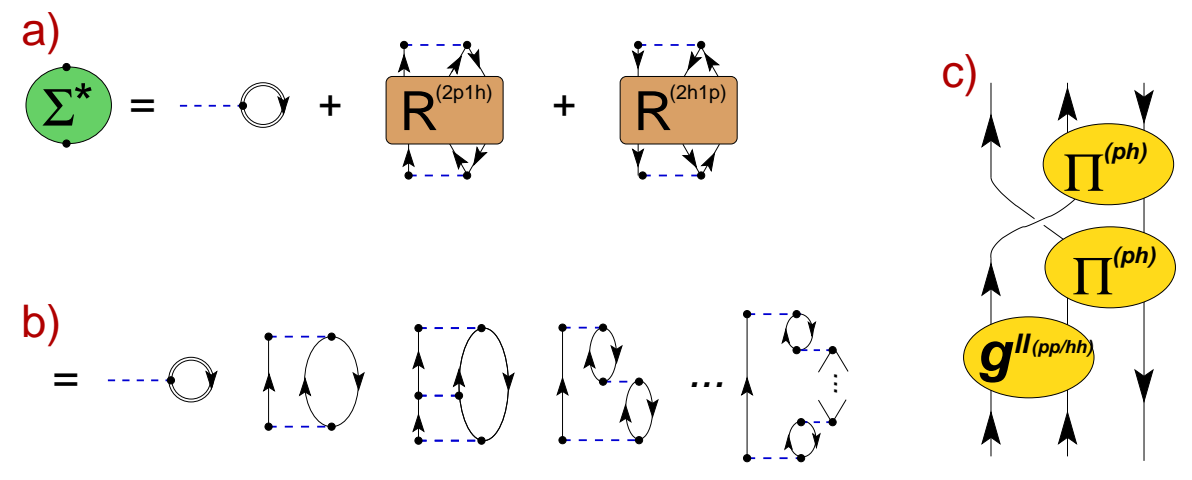

FIGURE 1. The self-energy $\Sigma^{\star}(\omega)$ separates exactly into a mean field term and the polarization propagators $R(\omega)$ for the $2 \mathrm{p} 1 \mathrm{~h} / 2 \mathrm{~h} 1 \mathrm{p}$ motion, as shown in a). The double line represent the correlated propagator of Eq. (1). Upon expansion of $R(\omega)$ in Feynman diagrams, one obtains the series of diagrams b) for the self-energy. The diagram c) gives an example of the contributions to $R^{2 p 1 h}(\omega)$ that are summed to all orders by the FRPA method.

where $\alpha, \beta, \ldots$, label a complete orthonormal basis set and $c_{\alpha}\left(c_{\beta}^{\dagger}\right)$ are the corresponding second quantization destruction (creation) operators. In these definitions, $\left|\Psi_{n}^{N+1}\right\rangle$, $\left|\Psi_{k}^{N-1}\right\rangle$ are the eigenstates, and $E_{n}^{N+1}, E_{k}^{N-1}$ the eigenenergies of the $(N \pm 1)$-electron system. Therefore, the poles of the propagator reflect the electron affinities and ionization energies. For a two-body hamiltonian, Eq. (1) also yields the total binding energy via the Migdal-Galitskiü-Koltun sum rule [6].

The one-body Green's function is computed by solving the Dyson equation

$$
g_{\alpha \beta}(\omega)=g_{\alpha \beta}^{0}(\omega)+\sum_{\gamma \delta} g_{\alpha \gamma}^{0}(\omega) \Sigma_{\gamma \delta}^{\star}(\omega) g_{\delta \beta}(\omega),
$$

where the irreducible self-energy $\Sigma_{\gamma \delta}^{\star}(\omega)$ acts as an effective, energy-dependent, potential. The latter can be expressed in terms of the exact propagator $g_{\alpha \beta}(\omega)$, which itself is a solution of Eq. (2), and the polarization propagator, $R(\omega)$, that accounts for deviations from the mean-field [7]. This is shown in Fig. 11a in terms of Feynman diagrams. The polarization propagator $R(\omega)$ is also expanded in terms of simpler propagators that involve the propagation of one quasiparticle [Eq. (1)] or more. This approach has the advantage to help identifing key physics ingredients of the many-body dynamics. By truncating to particular subsets of diagrams, one can then construct suitable approximations to the self-energy. Moreover, since infinite sets of linked diagrams are summed the approach is non-perturbative and satisfies the extensivity condition. This expansion also serves as guideline for systematic improvements of the method.

In the following we are interested in describing the coupling of $\mathrm{sp}$ motion to particlehole (ph) and two-particle (pp) or two-hole (hh) collective excitations of the system. Following Ref. [8], we first calculate the corresponding propagators by solving the random phase approximation (DRPA) equations in the $\mathrm{ph}$ and $\mathrm{pp} / \mathrm{hh}$ channels. These are then inserted in the self-energy by solving a set of Faddeev equations for the $R^{(2 p 1 h)}(\omega)$ and $R^{(2 h 1 p)}(\omega)$ propagators. Fig. 1c gives an example of one of the diagrams generated by this procedure. 
TABLE 1. Hartree-Fock and Faddeev-RPA binding energies (in Hartree) extrapolated from the cc-pVTZ and cc-pVQZ basis sets. The deviations from the experiment are indicated in parentheses (in $\mathrm{mH}$ ). For $\mathrm{Mg}$, the cc$\mathrm{pCV}(\mathrm{TQ}) \mathrm{Z}$ bases were used.

\begin{tabular}{cccc}
\hline & Hartree-Fock & FRPA & Experiment [12, 13] \\
\hline $\mathrm{He}$ & $-2.860(+44)$ & $-2.903(+1)$ & -2.904 \\
$\mathrm{Be}$ & $-14.573(+94)$ & $-14.643(+24)$ & -14.667 \\
$\mathrm{Ne}$ & $-128.547(+281)$ & $-128.917(+11)$ & -128.928 \\
$\mathrm{Mg}$ & $-199.617(+426)$ & $-200.058(-15)$ & -200.043 \\
\hline
\end{tabular}

The details of the Faddeev RPA (FRPA) approach are given in Refs. [8, 9]. For the present discussion it is sufficient to note that including only ph propagators corresponds to the same physics of the $G W$ [10] approach. This is known to give accurate binding energies for the electron gas, where the RPA is required to screen the long range Coulomb force. The FRPA method goes beyond the $G W$ since it accounts completely for Pauli correlations at the $2 \mathrm{p} 1 \mathrm{~h} / 2 \mathrm{~h} 1 \mathrm{p}$ level and include the propagation of $\mathrm{pp} / \mathrm{hh}$ configurations. The latter give crucial contribution to ionization energies in small systems [11].

Results. FRPA calculations were performed using the correlation consistent cc$\mathrm{pVTZ}$ and cc-pVQZ gaussian bases for all atoms except for the ground state of Mg. For the latter the core-valence version cc-pCV(TQ)Z were used, which include additional compact gaussians to improve the description of the core electrons. This choice was seen to improve the convergence of the binding energy for this atom. The results were then extrapolated to the basis set limit according to

$$
E_{X}=E_{\infty}+A X^{-3},
$$

where $X=T, Q$ is the cardinal number of the basis. This relation is known to give proper extrapolations for correlation energies. Here we apply it to ionization energies as well, remembering that these are also differences between eigenenergies. For the case of $\mathrm{Ne}$, this agrees well with the ionization energies obtained in a larger basis [9] (see below) and gives us confidence on the extrapolation procedure. We estimate that results given in Tabs. 1 and 2 are accurate within a few $\mathrm{mH}$.

Table 1 shows the results for the FRPA ground state energies and compares them to the experiment and the corresponding Hartree-Fock results. FRPA gives practically exact results for the two electron problem (He) while it accounts for $96 \%$ of the correlation energy in the larger systems. The atom of Be is an exception to this trend, due to the fact that this is not a good closed shell system. This leads to very soft excitations in the $\mathrm{J}^{\pi}=1^{-}, \mathrm{S}=1$ channel which can drive the ph RPA equation to instability. A proper treatment of this system may require improving the treatment of the excitation spectrum beyond the RPA.

Ionization energies are shown in Tab. 2. The extrapolated results deviate from experiment by about $5 \mathrm{mH}$ for the first ionization energies, while it increases to $10-15 \mathrm{mH}$ for the separation of slightly deeper electron orbits. The table also reports the predictions from Hartree-Fock theory and the second order self-energy (obtained by retaining just 
TABLE 2. Ionization energies obtained in Hartree-Fock, in second order perturbation theory for the self energy and with the full Faddeev-RPA (in Hartree). All results are extrapolated from the cc-pVTZ and cc-pVQZ basis sets. The deviations from the experiment are indicated in parentheses (in $\mathrm{mH}$ ).

\begin{tabular}{rcccc}
\hline & Hartree-Fock & $\mathbf{2}^{\text {nd }}$ order & FRPA & Experiment $[\mathbf{1 2}, \mathbf{1 3}]$ \\
\hline He: $1 \mathrm{~s}$ & $0.918(+14)$ & $0.906(+2)$ & $0.900(-4)$ & 0.904 \\
& & & & \\
$\mathrm{Be}: 2 \mathrm{~s}$ & $0.309(-34)$ & $0.320(-23)$ & $0.322(-21)$ & 0.343 \\
$1 \mathrm{~s}$ & $4.733(+200)$ & $4.620(+87)$ & $4.540(+7)$ & 4.533 \\
& & & & \\
$\mathrm{Ne}: 2 \mathrm{p}$ & $0.850(+57)$ & $0.763(-30)$ & $0.803(+10)$ & 0.793 \\
1s & $1.931(+149)$ & $1.750(-32)$ & $1.795(+13)$ & 1.782 \\
& & & & \\
$\mathrm{Mg}: 3 \mathrm{~s}$ & $0.253(-28)$ & $0.274(-7)$ & $0.277(-4)$ & 0.281 \\
2p & $2.281(+161)$ & $2.146(+26)$ & $2.130(+10)$ & 2.12 \\
& & & & \\
$\mathrm{Ar}: 3 \mathrm{p}$ & $0.590(+11)$ & $0.585(+6)$ & $0.578(-1)$ & 0.579 \\
3s & $1.276(+201)$ & $1.159(+84)$ & $1.065(-10)$ & 1.075 \\
2p & $9.570(+410)$ & $9.519(+359)$ & $9.199(+39)$ & 9.160 \\
\hline
\end{tabular}

the first two diagrams of Fig. 1 b). Second order corrections account for a large part of correlations but still lead to sizable errors. The additional correlations included in the present calculations appear to reduce this error substantially, in particular for deeper electron orbits. The importance of a treatment that is consistent with at least third order perturbation theory was already pointed out by Schirmer and co-workers in Ref. [11]. Such contribution were then included in the algebraic diagrammatic construction method at third order [ADC(3)] [14]. The present formulation of the FRPA includes the ADC(3) completely and gives similar results for small atomic systems. At the same time, the explicit inclusion of RPA phonons holds the promise for successful applications to extended systems. Further work will be required to verify that this is indeed the case.

The Ne atom was also computed in the FRPA approach by using a Hartree-Fock basis with a discretized continuum [9]. The size of the basis set was chosen large enough to approach the basis set limit. This gave first and second ionization energies of 0.801 and $1.795 \mathrm{H}$, in good agreement with Tab. 2. The total binding energy obtained is $128.888 \mathrm{H}$, somewhat in disagreement with the extrapolation from the cc-pV(TQ)Z bases and the experiment. The hole spectral function and momentum distribution of Ne are shown in Fig. 2. A moderate tail is generated at large momenta and originates from the $1 s$ orbit at $\approx-32 \mathrm{H}$. This is reproduced already at the Hartree-Fock level and it is due to the fact that the core electrons have the highest velocity in a $1 / r$ potential. We observe that this is a different situation to that of atomic nuclei, where high momentum components are seen at very large separation energies [15]. In the latter case the relevant strength is not found at fixed energies but distributed along a ridge in the energy-momentum plane with $E \approx k^{2} / 2 m$, which signals the presence of strong two-body correlations [7].

Conclusions. The Faddeev RPA method is an expansion of the many-body selfenergy that makes explicit the coupling between particle and collective phonons. First 

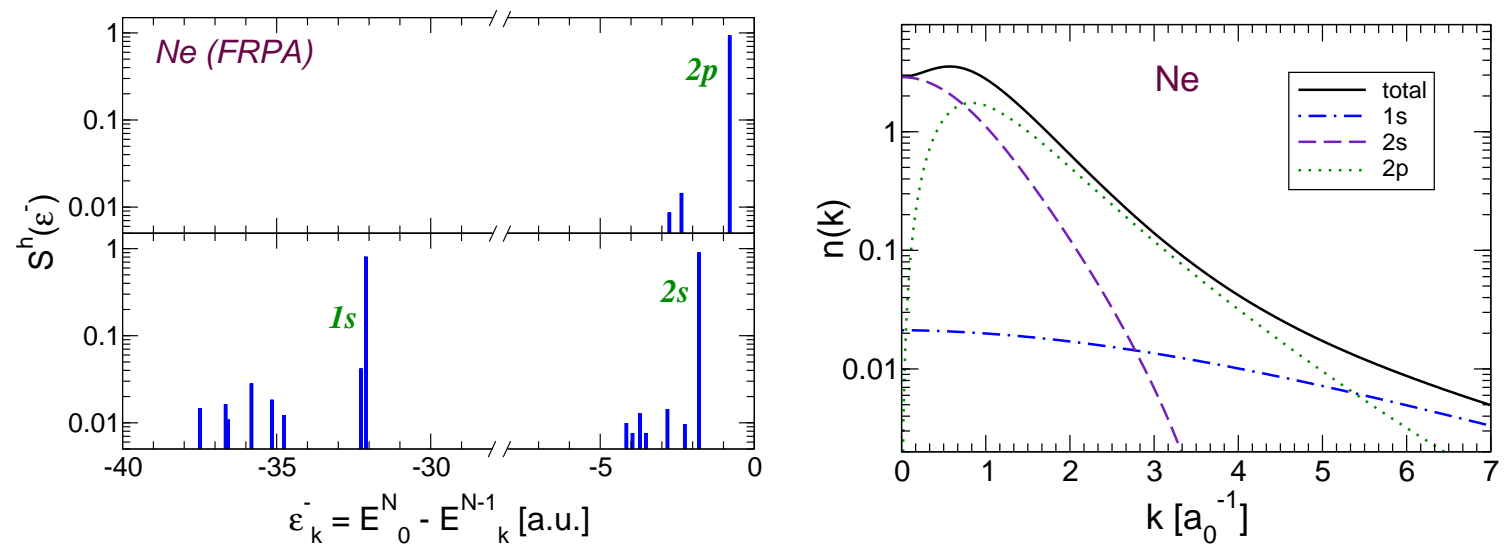

FIGURE 2. Hole spectral function (left) and momentum distribution (right) of the Ne atom. The dotted, dashed and dot-dashed lines are the contributions coming from the main $2 p, 2 s$ and $1 s$ quasihole peaks seen on the left side.

applications to small atoms have been reported in this talk and error for ionization energies were found to be of $\sim 10 \mathrm{mH}$. Accurate calculations of quasiparticle properties will serve in developments of the proposed QP-DFT [3]. Due to the inclusion of RPA, it is expected that the present FRPA formalism could reach similar accuracy also for extended systems. This will be the topic of future research efforts.

\section{ACKNOWLEDGMENTS}

We acknowledge several useful discussions with Prof. W. H. Dickhoff.

\section{REFERENCES}

1. W. Kohn and L. J. Sham, Phys. Rev. 140, A1133 (1965).

2. P. Hohenberg and W. Kohn, Phys. Rev. 136, B864 (1964).

3. D. Van Neck, S. Verdonck, G. Bonny, P. W. Ayers, and M. Waroquier, Phys. Rev. A 74, 042501(2006).

4. A. B. Migdal, Theory of Finite Fermi Systems and Applications to Atomic Nuclei (John Wiley and Sons, New York, 1967).

5. A. L. Fetter and J. D. Walecka, Quantum Theory of Many-Particle Physics (McGraw-Hill, New York, 1971).

6. W. H. Dickhoff and D. Van Neck, Many-Body Theory Exposed! (World Scientific, Singapore, 2005).

7. W. H. Dickhoff and C. Barbieri, Prog. Part. Nucl. Phys. 52, 377 (2004).

8. C. Barbieri and W. H. Dickhoff, Phys. Rev. C 63, 034313 (2001).

9. C. Barbieri, D. Van Neck and W. H. Dickhoff, Phys. Rev. A 76, 052503 (2007).

10. L. Hedin, Phys. Rev. 139, A796 (1965).

11. O. Walter and J. Schirmer, J. Phys. B:At. Mol. Phys. 14, 3805 (1981).

12. NIST Atomic Spectra Database, NIST Standard Reference Database \#78, http://physics.nist.gov/PhysRefData/ASD/in-dex.html

13. A. Thompson et al., X-ray Data Booklet (Lawrence Berkeley National Laboratory, Berkeley, CA, 2001), and references cited therein.

14. J. Schirmer, L.S. Cederbaum, and O. Walter, Phys. Rev. A 28, 1237 (1983)

15. D. Rohe, et al., Phys. Rev. Lett. 93, 182501 (2004). 